\title{
Biópsia Percutânea Estereotáxica no Diagnóstico das Lesões Mamárias Subclinicas
}

\author{
Core Biopsy for the Diagnosis of Subclinical Breast Lesions
}

\author{
Flávia Maria de Souza Clímaco, Euderson Kang Tourinho, \\ Aldo Franklin Ferreira Reis, Maria Inês Menescal Fabrício, \\ Maria de Fátima Ribeiro Maia, Jacir Luiz Balen, \\ Maria Consuelo Gondim Soares
}

\begin{abstract}
RESUMO
Objetivo: avaliar a eficácia da biópsia percutânea estereotáxica (BPE) no diagnóstico das lesões mamárias subclínicas, comparando seus resultados com a biópsia cirúrgica precedida de localização estereotáxica com fio guia.

Métodos: no periodo de janeiro de 1995 a fevereiro de 1997, realizamos um estudo transversal com 41 casos de lesões não-palpáveis em pacientes com idade superior a 35 anos, no Instituto de Ginecologia da Universidade Federal do Rio de Janeiro. Os casos foram classificados radiologicamente como benignos, provavelmente benignos, suspeitos e malignos. Para fins de análise estatística, as lesões benignas e provavelmente benignas foram estudadas em conjunto. O laudo histopatológico da BPE foi classificado em: inadequado para diagnóstico, ausência de malignidade, suspeito e maligno. O laudo histopatológico da biópsia cirúrgica foi classificado em: ausência de malignidade, pré-maligno e maligno. Calculamos a sensibilidade, a especificidade e os valores preditivos da BPE, assim como as razões de verossimilhança da mamografia e da BPE com a finalidade de predizer a probabilidade de câncer de mama.

Resultados: a BPE coincidiu com a biópsia cirúrgica em 86,2\% dos 29 casos de ausência de malignidade. Todos os casos suspeitos à BPE foram malignos à biópsia cirúrgica. Todos os casos de malignidade à BPE estiveram acordes com a biópsia cirúrgica. A sensibilidade e a especificidade da BPE foram 36,4\% e 100\%, respectivamente. O valor preditivo positivo do método foi $100 \%$ e o negativo $78,1 \%$. No grupo classificado como maligno à mamografia, a razão de verossimilhança foi 9,7, sendo 1,3 para os casos suspeitos e 0,1 para o laudo provavelmente benigno. A razão de verossimilhança da BPE foi infinita ( $\infty$ ) para as lesões suspeitas e malignas, 0,4 para os casos classificados como ausência de malignidade e 1,4
\end{abstract}

Instituto de Ginecologia da Universidade Federal do Rio de

Janeiro - RJ

Correspondência:

Flávia Maria de Souza Clímaco

R. Visconde de Pirajá, 525, apto. 403 - Ipanema

22410-003 - Rio de Janeiro - RJ 
para os casos inadequados para diagnóstico.

Conclusões: após análise dos resultados, por meio da razão de verossimilhança, concluimos que o laudo de ausência de malignidade pela BPE não nos permitiu afastar o diagnóstico de patologia maligna. Nestes casos, se não houver concordância entre o laudo histopatológico e o laudo mamográfico, deve-se prossseguir na investigação. Quando o laudo era suspeito à BPE, a probabilidade da lesão corresponder a carcinoma de mama foi muito alta. Deve-se proceder à biópsia cirúrgica para diagnóstico de certeza, pois o carcinoma infiltrante possui terapêutica diversa do carcinoma in situ e da hiperplasia atípica. Quando o laudo histopatológico era de malignidade, foi elevada a probabilidade da lesão corresponder a câncer e não observamos falso-positivos. Nestes casos, a BPE permite diagnóstico mais rápido, sem necessidade de biópsia cirúrgica.

PALAVRAS-CHAVE: Mama: câncer. Mamografia. Câncer: rastreamento.

\section{Introdução}

A mamografia é método de grande eficácia na detecção de lesões mamárias malignas clinicamente ocultas. Permite o diagnóstico do câncer de mama em estádio precoce, levando a tratamentos cirúrgicos mais conservadores e à redução da taxa de mortalidade. Com os recentes avanços no campo da mamografia, a técnica é reconhecida como o método de mais alta sensibilidade no rastreio de carcinomas nãopalpáveis, porém a sua relativa falta de especificidade é ainda um problema. Cresce, então, a necessidade de se realizarem procedimentos complementares invasivos para diagnóstico de certeza, seja por punção aspirativa por agulha fina para diagnóstico citológico, seja por biópsia percutânea ou biópsia cirúrgica após localização estereotáxica, para diagnóstico histopatológico.

A biópsia percutânea estereotáxica (BPE) é um dos métodos para o diagnóstico de pequenas lesões mamárias suspeitas de malignidade. Consiste na retirada de fragmentos de tecido da lesão impalpável para exame histopatológico. Foi primeiramente realizada por Parker et al. em $1990^{12}$ e, desde então, tem sido objeto de estudo por profissionais ligados à radiologia e à mastologia.

Para muitos, a BPE é considerada uma alternativa à biópsia cirúrgica, já que inúmeros trabalhos têm relatado alta precisão do método ${ }^{3,4,6,12,13}$. Apresenta vantagens em relação à biópsia cirúrgica: realização ambulatorial, melhor resultado estético, recuperação rápida e menor indice de infecção. Por produzir menor trauma, não acarreta lesões cicatriciais que possam levantar a suspeita de malignidade em mamografias posteriores. Apresenta, ainda, menor custo (em torno de US\$ 675) em relação à biópsia cirúrgica (varia entre US\$ 1,217 a US\$ 2,673) ${ }^{12}$, fato de extrema importância para os países em desenvolvimento, com escassos recursos financeiros aplicados na área de saúde. Além destas inúmeras vantagens, a utilização da BPE permite ao radiologista, auxiliado pelo patologista, fornecer ao médico assistente os elementos necessários para o planejamento terapêutico. Com isso, temos economia de tempo e redução significativa no número de biópsias cirúrgicas desnecessárias, principalmente nas lesões benignas.

Como observamos, por ser método de grande importância no diagnóstico de lesões mamárias detectadas pela mamografia, a BPE poderá ser utilizada nos programas de rastreio do câncer de mama como exame de $2^{\text {a }}$ linha. Por isso, iniciamos no Instituto de Ginecologia da Universidade Federal do Rio de Janeiro este trabalho visando avaliar a eficácia do método.

\section{Pacientes e Métodos}

No período de janeiro de 1995 a fevereiro de 1997, foram selecionadas 37 pacientes com idade superior de 35 anos, com exame clínico normal e que apresentavam lesões subclínicas observadas em mamografia realizada com a finalidade de rastreio do câncer de mama. As pacientes foram informadas sobre o exame que seria realizado, concordando em serem submetidas ao mesmo. Adotamos a classificação do American College of Radiology $^{2}$, que divide as imagens mamográficas 
em: benignas, provavelmente benignas, suspeitas ou com características de malignidade (malignas).

A inclusão no estudo obedeceu aos seguintes critérios: pacientes com imagem mamográfica suspeita ou maligna; pacientes com imagem mamográfica benigna ou provavelmente benigna, que sofreu alteração quer seja da forma, volume ou densidade durante o acompanhamento radiológico; pacientes com imagem mamográfica benigna ou provavelmente benigna que desejaram ser submetidas à biópsia ou que por alguma razão não poderiam ser acompanhadas pela mamografia. Para a análise estatística, as imagens mamográficas benignas foram agrupadas com as provavelmente benignas e estudadas em conjunto.

A ultra-sonografia de mama foi realizada para afastar cistos simples que não têm indicação de biópsia. Utilizamos mamógrafo de alta resolução (Mammomat 3 da Siemens S.A.) dotado de unidade para biópsia estereotáxica para realização do procedimento. Obedecemos à seguinte sistematização no que concerne à sua realização: instalação da unidade de estereotaxia ao mamógrafo e teste com Phanton para aferir a calibração do instrumento de estereotaxia.

Após estes cuidados, seguia-se o procedimento estereotáxico:

- Análise da mamografia visando rever a indicação de biópsia (Figura 1).

- Compressão da mama por placa de acrílico com janela central. Uma radiografia era realizada para observarmos se a lesão encontrava-se no centro da janela .

- Realizava-se radiografia em duas projeções variando a angulação em $16^{\circ}$, sendo informadas pelo computador as coordenadas da lesão.

- Em seguida, indicava-se o tamanho da agulha na unidade estereotáxica. Utilizamos agulha calibre 14, que era introduzida na mama devidamente acoplada à pistola automática. Antes da realização da biópsia, novas radiografias estereotáxicas eram feitas para verificação do posicionamento correto da agulha.

- Cinco disparos eram feitos, um no centro e os demais nas posições de 3, 6, 9 e 12 horas da lesão.

- Após o primeiro disparo, outra radiografia era realizada para avaliarmos o deslocamento da agulha através da lesão (Figura 2).

- Após a BPE, procedia-se à localização estereotáxica da lesão com fio guia. Colocava-se na pele um marcador radiopaco e duas radiografias, uma em incidência de perfil e outra em crâniocaudal (Figuras 3 e 4), eram realizadas. Assim, definia-se o ponto de entrada do fio na pele da mama e conseqüentemente a profundidade da lesão, permitindo ao cirurgião decidir sobre qual o melhor local da incisão cirúrgica para exérese da mesma.

- Nos casos de microcalcificações, os espécimes eram radiografados com técnica de ampliação, para observarmos se a biópsia fora eficaz (presença de microcalcificações nos fragmentos).

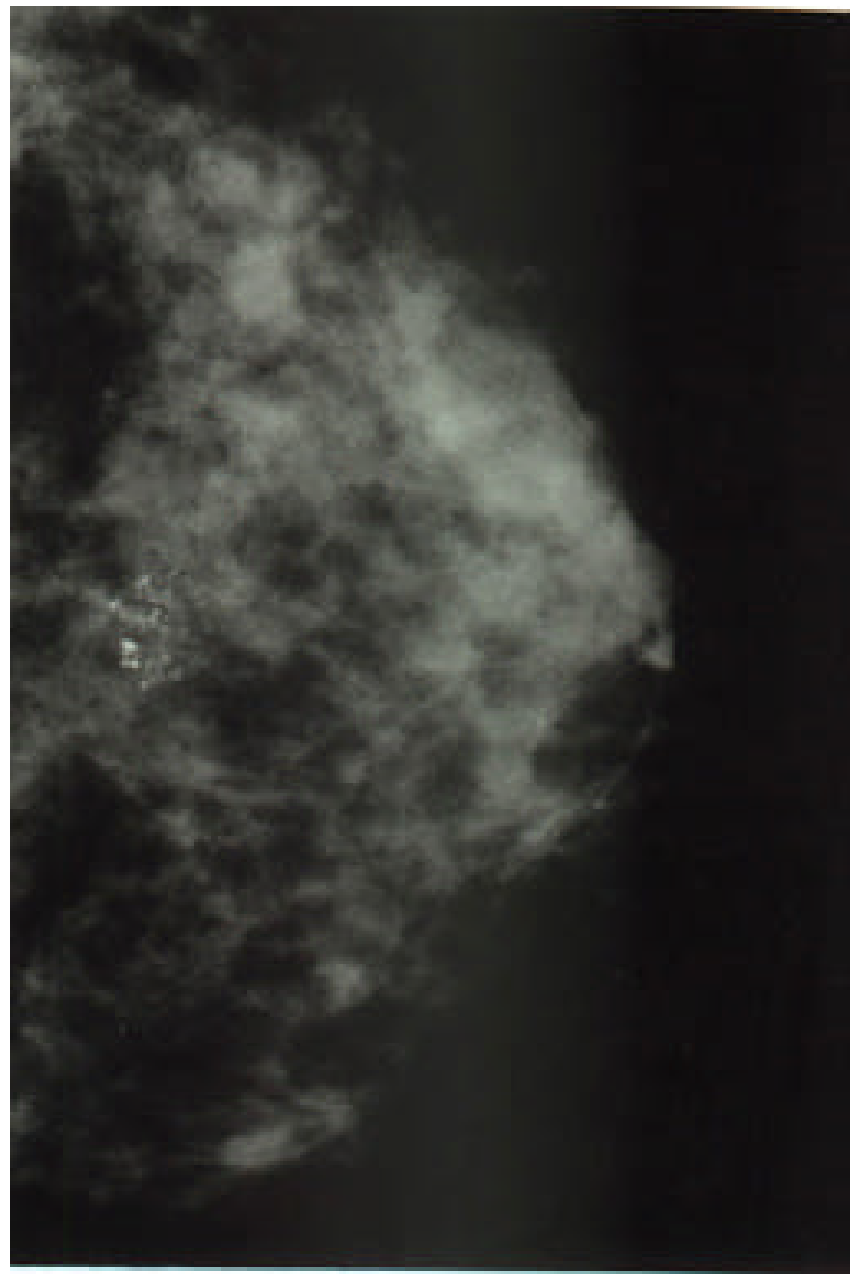

Figura 1 - Mamografia (incidência crânio-caudal) com microcalcificações agrupadas suspeitas.

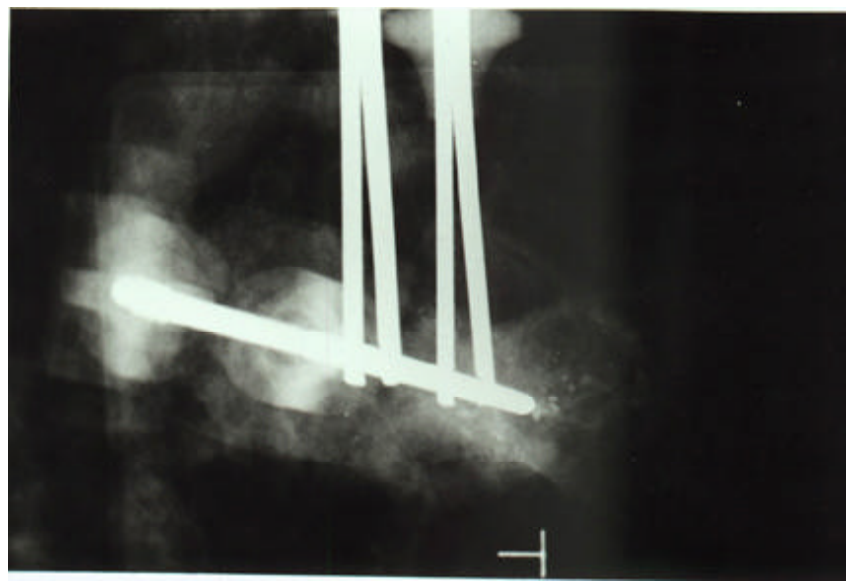

Figura 2 - Agulha de biópsia posicionada após o disparo. 


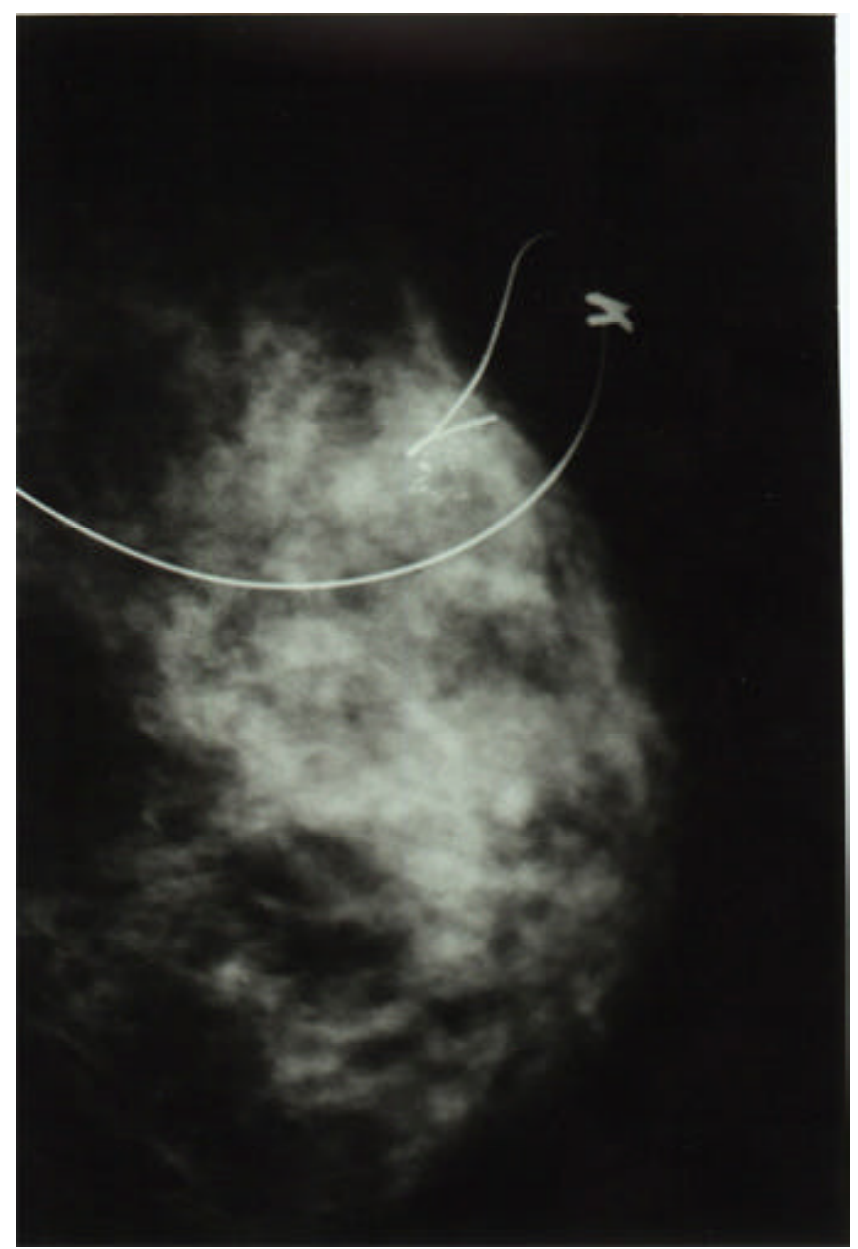

Figura 3 - Localização com fio de Kopans (incidência de perfil).

No dia seguinte, realizava-se biópsia cirúrgica sob anestesia geral obedecendo aos seguintes preceitos:

- Incisão cirúrgica próxima à área da lesão.

- Ressecção mais econômica quando nódulo.

- Ressecção ampla no caso de microcalcificações.

- Radiografia da peça cirúrgica para controle da exérese da lesão.

Classificamos o laudo histopatológico da BPE em: inadequado para diagnóstico (casos sem tecido mamário - epitélio ou tecido conjuntivo); ausência de malignidade; suspeito (hiperplasia epitelial atipica, carcinoma in situ e atipias celulares que não puderam ser determinadas) e maligno (carcinoma infiltrante). O laudo histopatológico da biópsia cirúrgica foi classificado em: ausência de malignidade; pré-maligno (hiperplasia epitelial atipica e carcinoma in situ) e maligno (carcinoma infiltrante). O carcinoma in situ foi definido como

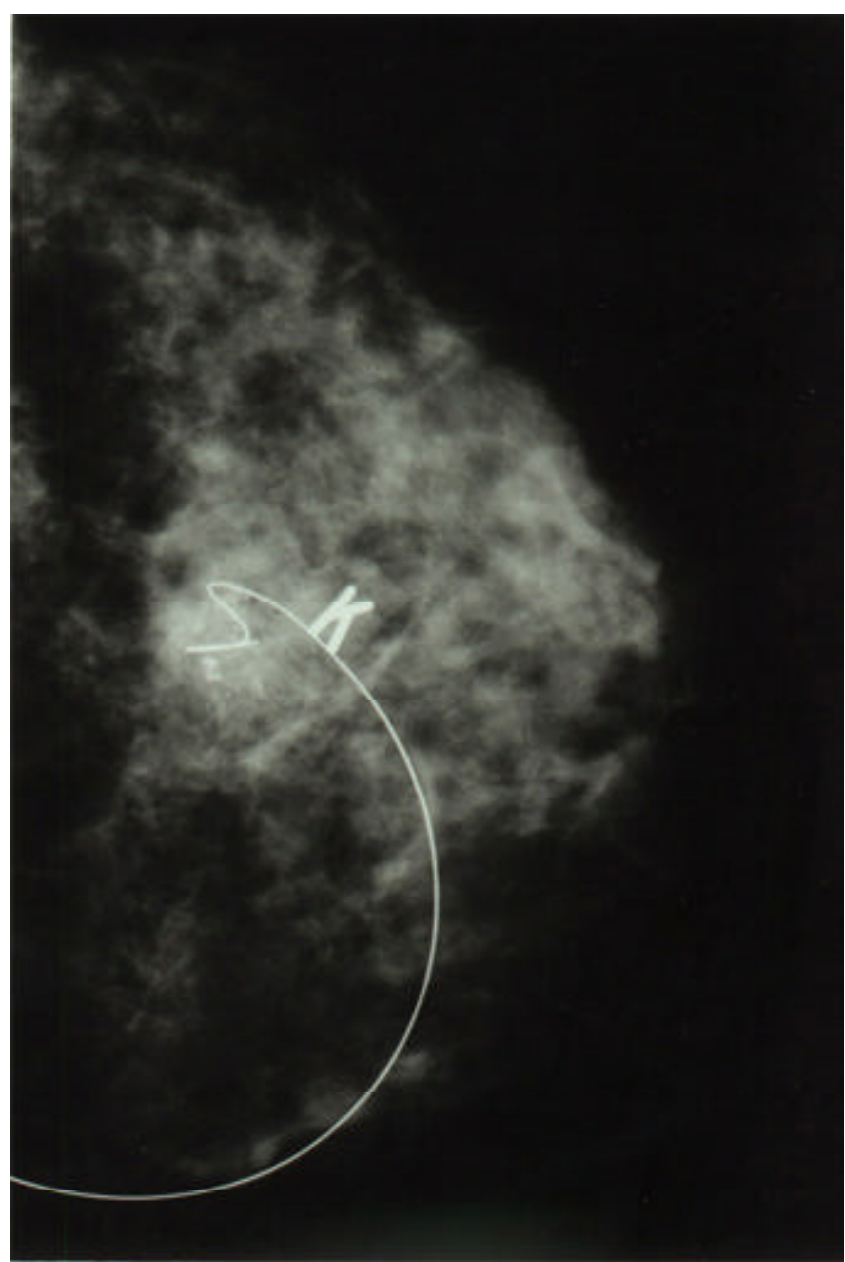

Figura 4 - Localização com fio de Kopans (incidência crânio-caudal).

lesão suspeita ou pré-maligna por não ter caráter de doença invasora como o carcinoma infiltrante.

Tanto os procedimentos estereotáxicos quanto a biópsia cirúrgica foram realizados por apenas um radiologista e um mastologista, respectivamente. $\mathrm{O}$ exame histopatológico da $\mathrm{BPE}$ foi feito cego em relação à biópsia cirúrgica e realizado por um patologista.

Os laudos da BPE e da biópsia cirúrgica foram comparados ao final do estudo. Calculamos a sensibilidade, a especificidade e os valores preditivos da BPE, assim como as razões de verossimilhança da mamografia e da BPE, com a finalidade de predizer a probabilidade de câncer de mama na amostra estudada.

O banco de dados utilizado foi o dBASE III Plus, versão 1.0. O processamento dos dados foi realizado usando o programa Epi Info (US Department of Health and Human Services and Public Health Service and Centers of Disease Control - USA) versão 5.01b. 
Resultados

A faixa etária das pacientes variou de 37 a 76 anos, com média de 54,2 anos e mediana de 53 anos. A média da dimensão mamográfica das lesões estudadas foi de 19,3 mm. Localizavam-se, preferencialmente, em quadrantes superiores $(70,7 \%)$, sendo que $48,8 \%$ das lesões estavam no quadrante súpero-externo, $14,6 \%$ no quadrante súpero-interno e $7,3 \%$ na intersecção dos quadrantes superiores. Cinco lesões $(12,2 \%)$ localizavam-se no quadrante ínfero-interno e as demais $(17,1 \%)$ estavam distribuídas por outros setores das mamas.

As imagens mamográficas corresponderam a formação nodular em 16 casos (39\%), a microcalcificações em 19 casos $(46,3 \%)$, a densidade assimétrica em dois casos (5\%), a formação nodular associada a microcalcificações em três casos $(7,3 \%)$ e a densidade assimétrica associada a microcalcificações em apenas um caso $(2,4 \%)$. A avaliação radiológica caracterizou 22 destas imagens $(53,7 \%)$ como provavelmente benignas, oito $(19,5 \%)$ como suspeitas e $11(26,8 \%)$ como malignas.

O diagnóstico histopatológico do material obtido pela BPE foi de ausência de malignidade em 29 casos $(70,7 \%)$, suspeito em $4(9,8 \%)$, maligno em $3(7,3 \%)$ e inadequado para diagnóstico em 5 casos $(12,2 \%)$. Dos casos inadequados para diagnóstico, 3 casos $(60 \%)$ correspondiam a microcalcificações e $2(40 \%)$ a nódulos.

O laudo histopatológico da biópsia cirúrgica foi de ausência de malignidade em 28 casos (68,3\%), pré-maligno em 2 (4,9\%) e maligno em 11 casos $(26,8 \%)$.

A Tabela 1 mostra a correlação entre os laudos histopatológicos obtidos pela BPE e pela biópsia cirúrgica.

Tabela 1 - Correlação entre os laudos histopatológicos da biópsia percutânea estereotáxica (BPE) e da biópsia cirúrgica.

\begin{tabular}{lcccc}
\hline BPE & Maligno & Pré-maligno & $\begin{array}{c}\text { Ausência } \\
\text { de malignidade }\end{array}$ & TOTAL \\
& $(\%)$ & $(\%)$ & - & 3 \\
& $3(100,0)$ & - & - & 4 \\
Maligno & $4(100,0)$ & - & $25(86,2)$ & 29 \\
$\begin{array}{l}\text { Suspeito } \\
\begin{array}{l}\text { Ausência de } \\
\text { Malignidade }\end{array}\end{array}$ & $2(6,9)$ & $2(6,9)$ & - & 5 \\
$\begin{array}{l}\text { Inadequado para } \\
\text { diagnóstico }\end{array}$ & $2(40,0)$ & - & & \\
\begin{tabular}{l} 
TOTAL \\
\hline
\end{tabular} & $\mathbf{1 1}(\mathbf{2 6 , 8 )}$ & $\mathbf{2 ( 4 , 9 )}$ & $\mathbf{2 ~ ( 4 , 9 )}$ & $\mathbf{4 1}$ \\
\hline
\end{tabular}

Para calcularmos a sensibilidade e a especificidade da BPE foi necessária a reclassificação dos laudos histopatológicos, tanto da BPE quanto da biópsia cirúrgica, em:

- Benigno, se o resultado fosse de ausência de malignidade, hiperplasia atípica, carcinoma lobular in situ ou atipias celulares que não puderam ser determinadas;

- Maligno, quando o diagnóstico histológico foi de carcinoma infiltrante ou carcinoma ductal in situ.

A Tabela 2 mostra a correlação entre os laudos histopatológicos da BPE e da biópsia cirúrgica, assim como a sensibilidade, a especificidade e os valores preditivos do método. Os casos inadequados para diagnóstico foram excluídos para fins desta análise estatística.

Tabela 2 - Correlação entre os laudos histopatológicos da biópsia percutânea estereotáxica (BPE) e da biópsia cirúrgica.

\begin{tabular}{lccc}
\hline & \multicolumn{2}{c}{ Biópsia cirúrgica } & \\
\cline { 2 - 3 } BPE & $\begin{array}{c}\text { Maligno } \\
\mathrm{n}(\%)\end{array}$ & $\begin{array}{c}\text { Pré-maligno } \\
\mathrm{n}(\%)\end{array}$ & TOTAL \\
\hline Maligno & $4(100,0)$ & - & $\mathbf{4}$ \\
Benigno & $7(21,9)$ & $25(78,1)$ & $\mathbf{3 2}$ \\
TOTAL & $\mathbf{1 1}(30,6)$ & $\mathbf{2 5}(\mathbf{6 9}, 4)$ & $\mathbf{3 6}$ \\
\hline
\end{tabular}

Sensibilidade $=36,4 \%$ $\mathrm{p}=0,0056022$

Especificidade $=100 \%$

Valor preditivo negativo $=78,1 \%$

Valor preditivo positivo $=100 \%$

Para o cálculo do risco de câncer conforme o laudo da BPE, primeiro foi necessário definirmos a probabilidade (prevalência) de câncer de mama por faixa etária (probabilidade pré-teste). Para isto utilizamos um estudo de base populacional realizado na cidade de Porto Alegre ${ }^{1}$. A Tabela 3 demonstra que a probabilidade de desenvolvimento de câncer de mama aumenta com a idade, principalmente a partir da quinta década. Observamos uma pequena diminuição da prevalência entre os 50 e 54 anos e 55 e 59 anos em relação à faixa etária de 45 a 49 anos, o que consideramos ser aleatória pelo pequeno número de casos de câncer por faixa etária .

Após a paciente ter se submetido à mamografia, calculamos por meio da razão de verossimilhança (RV) a probabilidade de câncer, por faixa etária, para cada laudo mamográfico (probabilidade pós-teste) (Tabela 3). Observamos que a probabilidade de uma paciente acima de 70 anos apresenter câncer de mama tendo mamografia com características de malignidade é 92 vezes maior 
que quando o laudo é provavelmente benigno. A probabilidade de câncer é aproximadamente 11 vezes maior nesta faixa etária, quando o laudo é maligno em comparação com a faixa etária dos 35 aos 39 anos.

Para o cálculo da RV pela BPE (Tabela 4), mantivemos a sua classificação histopatológica e reclassificamos os laudos da biópsia cirúrgica em benigno e maligno, como descrito anteriormente.

Tabela 3 - Probabilidade pós-teste de câncer de mama de acordo com o laudo da mamografia por faixa etária. RV = razão de verossimilhança.

Probabilidade pós-teste $(\%)$

\begin{tabular}{ccccc} 
Idade & Probabilidade & Maligno & Suspeito & $\begin{array}{c}\text { Provavelmente } \\
\text { benigno }\end{array}$ \\
(anos) & pré-teste (\%) & $\mathbf{( R V = 9 , 7 )}$ & $\mathbf{( R V = 1 , 3 )}$ & $\mathbf{( R V = 0 , 1 )}$ \\
\hline $35-39$ & 0,05 & 0,483 & 0,065 & 0,005 \\
$40-44$ & 0,06 & 0,579 & 0,078 & 0,006 \\
$45-49$ & 0,19 & 1,810 & 0,246 & 0,019 \\
$50-54$ & 0,16 & 1,528 & 0,208 & 0,016 \\
$55-59$ & 0,16 & 1,528 & 0,208 & 0,016 \\
$60-64$ & 0,21 & 1,996 & 0,272 & 0,021 \\
$65-69$ & 0,29 & 2,736 & 0,376 & 0,029 \\
$\geq$ a 70 & 0,56 & 5,152 & 0,723 & 0,056 \\
\hline
\end{tabular}

Tabela 4 - Razão de verossimilhança (RV) com a utilização da biópsia percutânea estereotáxica (BPE)

Biópsia cirúrgica

\begin{tabular}{lrrrr}
\cline { 2 - 3 } BPE & Maligno & Benigno & TOTAL & RV \\
\hline Maligno & 3 & - & $\mathbf{3}$ & $\infty$ \\
Suspeito & 4 & - & $\mathbf{4}$ & $\infty$ \\
Ausência de Malignidade & 4 & 25 & $\mathbf{2 9}$ & 0,4 \\
Inadequado para diagnóstico & 2 & 3 & $\mathbf{5}$ & 1,4 \\
TOTAL & $\mathbf{1 3}$ & $\mathbf{2 8}$ & $\mathbf{4 1}$ & - \\
\hline & & & & \multicolumn{3}{c}{$\mathrm{p}=0,0002$}
\end{tabular}

A seguir, as probabilidades pós-teste de acordo com o laudo da mamografia, por faixa etária, foram consideradas como probabilidades pré-teste, para o cálculo das probabilidades pós-testes com o uso da BPE no processo de diagnóstico da doença subclínica da mama. Ou seja, quando uma paciente se submete à mamografia, lesões subclínicas podem ser identificadas e estas têm sinais mamográficos de suspeição ou não, aumentando ou diminuindo a probabilidade da paciente ter câncer de mama. Quando estas lesões são biopsiadas, a probabilidade de câncer de mama pode aumentar ou diminuir de acordo com o laudo histopatológico.

A Tabela 5 relaciona as probabilidades pósteste de câncer de mama, por faixa etária e laudo mamográfico, calculadas para o laudo histopatológico de ausência de malignidade pela BPE. Neste caso, observamos que a probabilidade de câncer é aproximadamente duas vezes e meia menor que antes para qualquer laudo mamográfico.

Quando os laudos histopatológicos da BPE foram suspeitos ou malignos, a RV calculada foi infinita $(\infty)$, aumentando muito a probabilidade de câncer de mama para qualquer laudo mamográfico. Não calculamos a probabilidade pós-teste com laudo inadequado para diagnóstico, uma vez que a BPE neste caso não teve validade na elucidação do diagnóstico da doença, sendo necessária a realização de nova biópsia, seja ela percutânea ou cirúrgica.

\section{Discussão}

Apesar da BPE ser procedimento relativamente novo na propedêutica em Mastologia, inúmeros trabalhos comprovam que possui sensibilidade e especificidade elevadas ${ }^{3,4,5,6,10}$, sendo considerada de grande valor no diagnóstico precoce do câncer de mama.

Tabela 5 - Probabilidade pós-teste de câncer de mama, por faixa etária e laudo mamográfico, quando o laudo histopatológico da biópsia percutânea estereotáxica (BPE) é de ausência de malignidade $(\mathrm{RV}=0,4)$

\begin{tabular}{|c|c|c|c|c|c|c|}
\hline \multirow[b]{3}{*}{$\begin{array}{l}\text { Idade } \\
(\text { anos })\end{array}$} & \multicolumn{3}{|c|}{ Probabilidade pós-teste (\%) } & \multicolumn{3}{|c|}{ Probabilidade pós-teste $(\%)$} \\
\hline & \multicolumn{3}{|c|}{ Mamografia } & \multicolumn{3}{|c|}{ Mamografia } \\
\hline & Maligno & Suspeito & $\begin{array}{c}\text { Provavelmente } \\
\text { benigno }\end{array}$ & Maligno & Suspeito & $\begin{array}{c}\text { Provavelmente } \\
\text { benigno }\end{array}$ \\
\hline $35-39$ & 0,483 & 0,065 & 0,005 & 0,194 & 0,026 & 0,002 \\
\hline $40-44$ & 0,579 & 0,078 & 0,006 & 0,232 & 0,031 & 0,002 \\
\hline $50-54$ & 1,528 & 0,208 & 0,016 & 0,617 & 0,083 & 0,006 \\
\hline $55-59$ & 1,528 & 0,208 & 0,016 & 0,617 & 0,083 & 0,006 \\
\hline $60-64$ & 1,996 & 0,272 & 0,021 & 0,808 & 0,109 & 0,008 \\
\hline $65-69$ & 2,736 & 0,376 & 0,029 & 1,113 & 0,151 & 0,012 \\
\hline$\geq$ a 70 & 5,152 & 0,723 & 0,056 & 2,127 & 0,290 & 0,022 \\
\hline
\end{tabular}


Em nossa casuística, observamos sensibilidade de $36,4 \%$ e especificidade de $100 \%$. A taxa de falso-negativo foi, então, elevada (63,6\%). Achamos que a baixa sensibilidade ocorreu devido a menor experiência com o método, uma vez que começamos a utilizá-lo neste estudo, e também pelo pequeno número de casos para treinamento no procedimento.

A sensibilidade e a especificidade são propriedades de um teste e que devem ser consideradas para a tomada de decisão sobre pedilo ou não. Em outras palavras, são importantes na escolha do método diagnóstico diante de uma suspeita clinica. De posse do resultado do mesmo, a sensibilidade e a especificidade não mais terão importância. O dilema do clínico é saber se o paciente tem ou não a doença a partir dos resultados do teste.

A RV é uma forma alternativa de definir o desempenho de um método diagnóstico. A vantagem de sua utilização é que permite a análise de um teste sem que seja necessário dividir seu resultado em positivo (condição clínica presente) ou negativo (condição clínica ausente), como quando se utiliza a sensibilidade e especificidade. Pode ser usada para calcular a probabilidade de doença depois de um teste positivo, suspeito ou negativo (probabilidade pós-teste), ou seja, a RV estima a maior ou menor probabilidade de uma determinada doença estar presente em face de um determinado resultado.

Em nosso estudo, observamos que nos casos de ausência de malignidade pela BPE e laudo mamográfico provavelmente benigno, a probabilidade de câncer de mama diminuiu 2,5 vezes. Por haver concordância entre os laudos radiológico e histopatológico, as pacientes podem permanecer em controle radiológico.

Já nos casos de ausência de malignidade pela BPE e laudo mamográfico suspeito ou maligno, a probabilidade de câncer também diminuiu 2,5 vezes, porém permanecia respectivamente 12 a 16 vezes e 92 a 116 vezes maior quando comparada ao laudo provavelmente benigno. Pelo risco de câncer de mama permanecer elevado e por não haver concordância entre os laudos mamográfico e histopatológico, devemos prosseguir na investigação realizando biópsia cirúrgica.

O laudo suspeito à BPE (hiperplasia atípica, carcinoma in situ e casos com atipias celulares que não puderam ser determinadas) aumentou de maneira significativa a probabilidade de câncer de mama. Contudo, nestes casos, deve-se proceder à biópsia cirúrgica para diagnóstico de certeza, pois o carcinoma infiltrante possui terapêutica diversa destas lesões. Sabemos que existe a possibilidade de lesões atípicas e carcinomas in situ se localizarem na periferia de um carcinoma infiltrante. Para Liberman et al. ${ }^{8,9}$, o achado de hiperplasia atípica à BPE é indicação para a biópsia cirúrgica por causa da alta prevalência (52\%) de carcinoma nestas lesões e por ser o diagnóstico diferencial com doença maligna difícil, principalmente quando pequenas áreas estão envolvidas.

Nos casos em que o laudo da BPE foi de malignidade (carcinoma infiltrante), a RV foi infinita $(\infty)$ e o diagnóstico de malignidade firmado, já que não observamos resultados falso-positivos em nosso estudo. Nestes casos, a conduta terapêutica pode ser decidida sem a necessidade de realização de biópsia cirúrgica, reduzindo os custos e a demora no diagnóstico.

Apesar de termos calculado a RV para o laudo inadequado para diagnóstico, não há sentido em calcularmos a probabilidade pós-teste, já que neste caso se deve prosseguir na investigação realizando nova biópsia percutânea ou, de preferência, biópsia cirúrgica. Em nosso estudo, dos cinco casos inadequados para diagnóstico, a maioria (60\%) correspondeu a microcalcificações e apenas cinco fragmentos foram retirados por exame.

Liberman et al. ${ }^{7}$ preconizam que cinco fragmentos devem ser obtidos caso a lesão em estudo seja uma formação nodular, sendo possivel o diagnóstico histopatológico. Para microcalcificações, no minimo cinco fragmentos são retirados inicialmente e, em seguida, a radiografia do espécime é realizada. Se microcalcificações não forem radiologicamente identificadas, fragmentos adicionais são obtidos até que as mesmas sejam observadas. Em nosso estudo, quando não observávamos microcalcificações na radiografia, a obtenção de mais fragmentos pela BPE não era possivel, já que o controle radiológico dos mesmos era feito ao término da localização da lesão com fio guia e utilizamos apenas um mamógrafo, tanto para realização do procedimento estereotáxico quanto para a radiografia dos fragmentos da biópsia percutânea. Achamos também que a utilidade deste método no diagnóstico das microcalcificações não está tão bem estabelecida como para o diagnóstico das formações nodulares. Parker ${ }^{11}$ recomenda obtenção de no mínimo 10 fragmentos nos casos de microcalcificações, pois há maior ocorrência de resultados falso-negativos. Em nossa casuística, o número de lesões com microcalcificações foi elevado (46,3\% dos casos). Este dado, associado ao controle radiológico após o término do procedimento, foi talvez o responsável pelo maior número de laudos inadequados para diagnóstico.

Não foi possivel comparar os resultados obtidos com a RV da BPE, por não existirem trabalhos na literatura avaliando o método por meio 
deste estudo estatístico e pelo fato dos diversos autores não classificarem o laudo histopatológico como o fizemos, impossibilitando que fizéssemos o cálculo da RV.

\section{SUMMARY}

Purpose: to evaluate core biopsy (CB) for the diagnosis of subclinical breast lesions, comparing with surgical biopsy previously identified by stereotaxic mammography.

Methods: this is a cross-sectional study of 41 subclinical lesion cases over 35 years of age, between January 1995 and February 1997 at the Instituto de Ginecologia da Universidade Federal do Rio de Janeiro. The cases were classified mammographically as benign, probably benign, suspicious and malignant. Benign and probably benign lesions were studied together for statistical purposes. The histopathologic diagnosis of $C B$ was classified as inadequate for diagnosis, absence of malignancy, suspicious and malignant. The histopathologic diagnosis of the surgical biopsy was classified as absence of malignancy, pre-malignant and malignant. The sensitivity, especificity and predictive values of $C B$ were evaluated. Mammography likelihood ratio and core biopsy likelihood ratio were evaluated to predict breast cancer.

Results: $C B$ coincided with surgical biopsy in $86.2 \%$ of the 29 cases of absence of malignancy. All cases suspected by $C B$ were malignant by surgical biopsy. All cases malignant by $C B$ were also malignant by surgical biopsy. $C B$ sensitivity and specificity were $36.4 \%$ and $100 \%$, respectively. Positive predictive value was $100 \%$ and negative predictive value was $78.1 \%$. In the group classified mammographically as malignant the likelihood ratio was 9.7; for suspicious lesions it was 1.3 and for probably benign lesions it was 0.1. Core biopsy likelihood ratio was infinite (¥) for suspicious and malignant lesions, 0.4 for cases classified as absence of malignancy and 1.4 for inadequate for diagnosis cases.

Conclusions: after analysis of the results, with the use of the likelihood ratio, we conclude that $C B$ report of absence of malignancy did not allow to rule out the diagnosis of malignancy. In these cases, we should correlate the result with mammography. If CB shows absence of malignancy and there is no correlation with mammography, the research must be continued. When the report of $C B$ was suspicious, the probability of a breast carcinoma was very high. In these cases, we should perform a surgical biopsy to establish a definitive diagnosis, because an infiltrating carcinoma needs a different therapy when compared with carcinoma in situ and atypical hyperplasia. In the cases of histopathologic report of malignancy, the probability of breast cancer was high, since we did not observe any false positive $C B$. In these cases, $C B$ allowed a quick diagnosis without the need of surgical biopsy.

KEY WORDS: Breast neoplasm. Cancer: screening. Mammography.

\section{Referências}

1. Brasil. Ministério da Saúde. Secretaria Nacional de Assistência à Saúde. Instituto Nacional do Cancer (INCa) - Coordenação de Programas de Controle de Câncer (Pro-Onco). Câncer no Brasil - dados dos registros de base populacional - volume II. Rio de Janeiro, 1995. v. 2, 88p.

2. Brenner JR, Fajardo L, Fisher PR, Dershaw DD, Evans WP, Basset L, et al. Percutaneous core biopsy of the breast: effect of operator experience and number of samples on diagnostic accuracy. Am J Roentgenol 1996; 166: 341-6.

3. Caines JS, McPhee MD, Konok GP, Wright BA. Stereotaxic needle core biopsy of breast lesions using a regular mammographic table with an adaptable stereotaxic device. Am J Roentgenol 1994; 163: 317-21.

4. Doyle AJ, Murray KA, Nelson EW, Bragg DG. Selective use of image-guided large core needle biopsy of the breast: accuracy and cost-effectiveness. Am J Roentgenol 1995; 165: 281-4.

5. Elvecrog EL, Lechner MC, Nelson MT. Nonpalpable breast lesions: correlation of stereotaxic large-core needle biopsy and surgical biopsy results. Radiology 1993; 188: 453-5.

6. Janes RH, Bouton MS. Initial 300 consecutive stereotactic core needle breast biopsies by a surgical group. Am J Surg 1994; 168: 533-7.

7. Liberman L, Dershaw DD, Rosen PP, Abramson AF, Deutch BM, Hann LE. Stereotaxic 14-gauge breast biopsy: how many core biopsy specimens are needed? Radiology 1994; 192: 793-5.

8. Liberman L, Cohen MA, Dershaw DD, Abramson AF, Hann LE, Rosen PP. Atypical ductal hiperplasia diagnosed at stereotaxic core biopsy of breast lesions: an indication of surgical biopsy. Am J Roentgenol 1995; 164: 1111-3.

9. Liberman L, Fahs MC, Dershaw DD, Bonaccio E, Abramson AF, Cohen MA, et al. Impact of stereotaxic core breast biopsy on cost of diagnosis. Radiology 1995; 195: 633-7.

10.Nath ME, Robinson TM, Tobon H, Chough DM, Sumkin JH. Automated large-core needle biopsy of surgically removed breast lesions: comparison of samples obtained with 14-, 16-, e 18-gauge needles. Radiology 1995; 197: 739-42.

11.Parker SH. Percutaneous large core breast biopsy. Cancer 1994; 74: 256-62.

12.Parker SH, Lovin JD, Jobe WE, Luethke JM, Hopper KD, Yakes WF, et al. Stereotactic breast biopsy with a biopsy gun. Radiology 1990; 176: 741-7.

13.Parker SH, Burbank F, Jackman RJ, Aucreman CJ, Cardenosa G, Cink TM, et al. Percutaneous largecore breast biopsy - a multi-institutional study. Radiology 1994; 193: 359-64. 